\title{
ON THE ORLICZ SYMMETRY OPERATOR
}

\author{
LUJun GuO AND RUIFANG CHEN
}

Abstract. R. Schneider (1970) proved that if $K \in \mathbb{R}^{n}$ is a convex body, such that each shadow boundary of $K$ with respect to parallel illumination halves the Euclidean surface area of $K$, then $K$ is centrally symmetric. A generalization of the results of R. Schneider was given by G. Averkov, E. Makai and H. Martini (2009). In this paper, by introducing an Orlicz symmetry operator $\Delta_{\phi}: \mathscr{K}^{n} \rightarrow \mathscr{K}^{n}$, we show a new method to obtain the characterization of symmetry for convex bodies. As an application, we will show that there is a unique member of $\Delta_{\phi}\langle K\rangle$ characterized by having larger volume than that of any other member of $\Delta_{\phi}\langle K\rangle$, where $\Delta_{\phi}\langle K\rangle$ is the Orlicz symmetric equivalence class of $K$.

Mathematics subject classification (2010): 52A20, 52A40, 33C55.

Keywords and phrases: Central symmetry, Minkowski space, normed linear space, shadow boundary, Steiner symmetrization, surface area measure.

\section{REFERENCES}

[1] G. Averkov, E. MaKai And H. Martini, Characterizations of central symmetry for convex bodies in Minkowski spaces, Studia Sci. Math. Hungar. 46 (2009), 493-514.

[2] F. Chen, J. Zhou, And C. YAng, On the reverse Orlicz Busemann-Petty centroid inequality, Adv. Appl. Math. 47 (2011), 820-828.

[3] R. Gardner, Geometric Tomography, second ed., Encyclopedia Math. Appl., vol. 58, Cambridge University Press, Cambridge, 2006.

[4] R. J. Gardner, D. Hug, AND W. Weil, The Orlicz-Brunn-Minkowski theory: A general framework, additions, and inequalities, J. Differential Geom., 97 (2014), 427-476.

[5] L. Guo, C. Du And G. Leng, On the Orlicz zonoid operator, J. Math. Anal. Appl., 424 (2015), 1261-1271.

[6] C. Haberl, E. Lutwak, D. Yang, And G. Zhang, The even Orlicz Minkowski problem, Adv. Math. 224 (2010), 2485-2510.

[7] C. Haberl and F. E. Schuster, General $L_{p}$ affine isoperimetric inequalities, J. Differential Geom. 83 (2009), 1-26.

[8] C. Haberl and F. E. Schuster, Asymmetric affine $L_{p}$ Sobolev inequalities, J. Funct. Anal. 257 (2009), 641-658.

[9] C. Haberl, F. E. Schuster And J. XIaO, An asymmetric affine Pólya-Szegö principle, Math. Ann. 352 (2012), 517-542.

[10] Q. Huang And B. He, On the Orlicz Minkowski problem for polytopes, Discrete Comput. Geom. 48 (2012), 281-297.

[11] A. J. Li AND G. S. Leng, A new proof of the Orlicz Busemann-Petty centroid inequality, Proc. Amer. Math. Soc. 139 (2011), 1473-1481.

[12] M. Ludwig, Minkowski valuations, Trans. Amer. Math. Soc. 357 (2005), 4191-4213.

[13] M. Ludwig, Minkowski areas and valuations, J. Differential Geom. 86 (2010), 133-161.

[14] M. Ludwig, General affine surface areas, Adv. Math. 224 (2010), 2346-2360.

[15] M. Ludwig And M. Reitzner, A classification of $S L(n)$ invariant valuations, Ann. of Math. 172 (2010), 1223-1271.

[16] E. LutwaK, The Brunn-Minkowski-Firey theory I: Mixed volumes and the Minkowski problem, J. Differential Geom. 38 (1993), 131-150. 
[17] E. Lutwak, D. Yang, And G. Zhang, Orlicz centroid bodies, J. Differential Geom. 84 (2010), $365-387$.

[18] E. Lutwak, D. YAng, And G. Zhang, Orlicz projection bodies, Adv. Math. 223 (2010), 220-242.

[19] L. ParapatiTs, $S L(n)$-covariant $L_{p}$-Minkowski valuations, J. Lond. Math. Soc., in press.

[20] L. Parapatits, $S L(n)$-contravariant $L_{p}$-Minkowski valuations, Trans. Amer. Math. Soc., in press.

[21] R. SCHNEIDER, Über eine Integralgleichung in der Theorie der konvexen Körper, Math. Nachr. 44 (1970), 55-75.

[22] R. SchneIder, Curvature measures of convex bodies, Ann. Mat. Pura Appl. 116 (1978), 101-134.

[23] R. Schneider, Convex Bodies: The Brunn-Minkowski Theory, Encyclopedia Math. Appl., vol. 44, Cambridge University Press, Cambridge, 1993.

[24] F. E. SChUSter AND M. Weberndorfer, Volume inequalities for asymmetric Wulff shapes, J. Differential Geom. 92 (2012), 263-283.

[25] G. T. WAng, G. S. Leng AND Q. Z. HuAng, Volume inequalities for Orlicz zonotopes, J. Math. Anal. Appl. 391 (2012) 183-189.

[26] M. Weberndorfer, Shadow systems of asymmetric L L zonotopes, Adv. Math. 240 (2013), 613635.

[27] Dongmeng Xi, Hailin Jin and Gangsong Leng, The Orlicz Brunn-Minkowski inequality, Adv. Math. 260 (2014), 350-374.

[28] G. ZHU, The Orlicz centroid inequality for star bodies, Adv. in Appl. Math. 48 (2012), 432-445. 